\title{
Método de pós-fechamento do Balanço de Energia para avaliação dos fluxos de modelos da superfície terrestre.
}

Post-Closure of the Energy Balance method to evaluate of the fluxes of the land surface models

Fabíola Carolina Pereira Valente ${ }^{1}$, Jônatan Dupont Tatsch², Débora Regina Roberti ${ }^{3}$,

Claudio Alberto Teichrieb ${ }^{4}$, Marcelo Bortoluzzi Diaz ${ }^{5}$, Gisele Cristina Dotto Rubert ${ }^{6}$

e Daniele Morgenstern Aimi ${ }^{7}$

\author{
${ }^{1}$ Mestranda em Meteorologia, Universidade Federal de Santa Maria, Santa Maria, RS, Brasil. \\ 2,3 Professor Adjunto do Departamento de Física, Universidade Federal de Santa Maria, Santa Maria, RS, Brasil. \\ ${ }^{4}$ Bolsista Pós Doutoral, Universidade Federal de Santa Maria, Santa Maria, RS, Brasil. \\ ${ }^{5,6}$ Doutorando em Meteorologia, Universidade Federal de Santa Maria, Santa Maria, RS, Brasil. \\ 7 Doutoranda em Física, Universidade Federal de Santa Maria, Santa Maria, RS, Brasil. \\ fabiolavalentepereira@gmail.com; jdtatsch@gmail.com; debora@ufsm.br; teichrieb@gmail.com; marbdiaz@gmail.com; \\ girubert@gmail.com; danielefm@gmail.com
}

\begin{abstract}
Resumo
Neste trabalho foi feita uma análise da incerteza dos fluxos de energia resultantes dos métodos pós-fechamento do Balanço de energia: Método H, Método LE e Método Bowen para os dados observados em área de campo nativo do bioma Pampa, localizado em Santa Maria, RS, Brasil . A partir destes três métodos sugerimos um novo, ao qual chamamos de Método Combinado. Este método leva em conta as classes de qualidade dos fluxos turbulentos para a escolha do método de correção a ser aplicado. As incertezas dos fluxos foram obtidas pela diferença entre os fluxos corrigidos por um dos métodos pós-fechamento e os fluxos brutos. Os valores máximos das incertezas ocorrem no período diurno. Quanto ao Método Combinado, a maior porcentagem de casos resultou na aplicação da correção pelo Método Bowen, seguido do Método LE e com menos ocorrência o Método H. Dessa forma, este Método pode ser considerado válido para avaliar a incerteza dos fluxos simulados por modelos de superfície terrestre.
\end{abstract}

Palavras-chave: Balanço de energia, fluxos turbulentos, incerteza dos fluxos, Métodos pós-fechamento, Método Combinado.

\begin{abstract}
In this work, an analysis of the uncertainty of energy flux resulting from post-closure methods of the energy balance: H Method, LE method and Bowen method for the data observed in native grass area of the Pampa biome, located in Santa Maria, RS, Brazil. From these three methods suggest a new one, which we call the Combined Method. This method takes into account the quality classes of eddy covariance to choose the correct method to be applied. The uncertainties of flux were obtained by the difference between the fluxes corrected by one of the postclosure methods and the raw fluxes. The maximum values of the uncertainties occur during the daytime. Regarding the combined method, the highest percentage of cases resulted in the application of correction by Bowen method, followed by the LE method and with less occurrence Method H. Thus, this method can be considered valid to evaluate the uncertainty of simulated fluxes over land surface models.
\end{abstract}

Keywords: Energy balance, eddy covariance, uncertainty fluxes, post-closure methods, Combined Method. 


\section{Introdução}

técnica eddy covariance tem sido nas últimas décadas a ferramenta mais utilizada para o monitoramento das trocas de fluxos de energia e massa entre a biosfera e a atmosfera (Baldocchi et al., 1988).

A acurácia do método é feita pelo fechamento do balanço de energia ( $\mathrm{Rn}-\mathrm{G}=\mathrm{H}+\mathrm{LE}$ ), em que Rn é o saldo de radiação, $G$ o fluxo de calor no solo, H é o fluxo de calor sensível e LE o fluxo de calor latente. Os termos $\mathrm{H}$ e LE são obtidos pelo cálculo da covariância da componente vertical do vento e o escalar de interesse (temperatura do ar para $\mathrm{H}$ e umidade do ar para LE). O grau de fechamento é expresso pela Razão do Balanço de Energia $(\mathrm{RBE}=\mathrm{H}+\mathrm{LE} / \mathrm{Rn}-\mathrm{G})$.

Geralmente, a componente H + LE subestima os fluxos de 10-30\% (Wilson et al., 2002), sendo assim o imbalanço de energia um problema em aberto na Micrometeorologia e que tem sido tema de muitas discussões e pesquisas acerca do entendimento das possíveis causas dessa falha (Foken et al. 2011, Leuning et al., 2012).

Os fluxos medidos pelo método eddy covariance são usados para calibrar e validar modelos de superfície. No entanto, em virtude das incertezas dos fluxos medidos são usados métodos de ajustes pós-fechamento. $\mathrm{O}$ método mais usado é o Razão de Bowen (Método Bowen) em que o resíduo do balanço de energia é particionado para $\mathrm{H}$ e LE de acordo com o valor medido (Twine et al., 2000). Outros dois métodos menos usados são o Método LE (H) que atribui o resíduo integralmente a LE $(\mathrm{H})$.

Neste artigo propõe-se um método de pósfechamento baseado na combinação dos métodos anteriores, onde o critério de escolha do método é definido de acordo com a classe de qualidade dos fluxos (mais detalhes na seção 2.3). Esse método pode ser usado para avaliar a incerteza dos fluxos simulados por modelos de superfície terrestre.

\section{Materiais e métodos}

\subsection{Descrição da Área Experimental}

O sítio experimental fica localizado em área pertencente à Universidade Federal de
Santa Maria - UFSM (Latitude: -29,7241; Longitude: -53,7597; Altitude: 88 m. A região é coberta por campo nativo do bioma Pampa. O clima de Santa Maria, de acordo com a classificação de Köppen é do tipo Cfa, com precipitação média anual em torno de 1650 $\mathrm{mm}$, bem distribuída ao longo do ano e temperatura anual média de $19,5{ }^{\circ} \mathrm{C}$ (Roberti et al., 2013).

A torre micrometeorológica da rede SULFLUX, instalada no sítio de estudo é instrumentada por um Sistema Eddy Covariance (EC) instalado à 3 metros de altura, consistindo de um Analisador de Gás Infravermelho de caminho fechado (LI 7200, LICOR) e um anemômetro sônico 3D (Wind Master Pro, Gill Instruments), com amostragens realizadas em 10 $\mathrm{Hz}$, um saldo radiômetro (CNR4, Kipp \& Zonnen, 3 metros de altura), um termohigrômetro (HMP155, Vaisala Inc., USA, 3 metros de altura), um pluviômetro (TR525USW, Texas electronics, Inc., posicionado à 6 metros de altura). Em volta da torre foram instalados três fluxímetros (HFP01, Hukseflux), três termômetros de solo (modelo 108, Campbell Scientific Inc., UK) e três TDR para medir o conteúdo de água no solo (Theta probe type ML2x, Delta-T Device) em que estes sensores de solo foram colocados em três pontos horizontalmente distintos e posicionados $5 \mathrm{~cm}$ abaixo do solo.

\subsection{Processamento dos dados}

Os dados brutos EC foram processados por um software comercial (EddyPro®, V 5.1.1 - LiCor, Lincon, Nebraska, USA). Para o cálculo e correção dos fluxos de energia e massa foi utilizada a seguinte configuração: flutuações turbulentas em torno de média móvel de 30 minutos, flutuações de densidade (Webb et al., 1980), rotação de coordenadas dupla (Wilczak et al., 2001), maximização da covariância (padrão) para a compensação da defasagem de tempo entre os sensores, filtro passa alta (lbrom, 2007) e baixa (Moncrieff et al., 2004), testes de controle de qualidade dos fluxos (Foken et al., 2004). 


\subsection{Incerteza dos Métodos de pós- fechamento}

A incerteza dos métodos é uma forma de representar possíveis erros sistemáticos dos fluxos turbulentos devido ao desconhecimento da causa do imbalanço de energia (Ingwersen et al., 2015).

Uma banda ou intervalo de incerteza pode ser obtido pela diferença entre os fluxos ajustados por um dos métodos de pósfechamento e os fluxos brutos. Os fluxos brutos de $\mathrm{H}$ (LE) são equivalentes aos fluxos corrigidos pelo Método LE $(\mathrm{H})$.

Assim, para a avaliação das incertezas, foram adotados quatro métodos de pós-fechamento do balanço de energia. Destes, propomos o Método Combinado, em que a escolha do método a ser aplicado para a correção dos fluxos depende da classe de qualidade dos fluxos (qc_H e qc_LE).

Estas classes de controle de qualidade variam de 1 a 9 e são determinados por critérios de estacionariedade e de turbulência desenvolvida, em que 1 representa a melhor qualidade e 9 a pior (Foken et al., 2004). Foi feito um reagrupamento destas classes de modo que as classes 1 a 3 foram rotuladas como classe 1 (melhor qualidade), classes 4 a 6, rotuladas como classe 2 (boa qualidade), classes 7 e 8, rotuladas como classe 3 (qualidade moderada) e os da classe 9 foram desprezados.

Após esta reclassificação, o método de correção foi definido da seguinte forma:

- qc_H = qc_LE, aplica-se o Método Bowen;

- qc_H > qc_LE, aplica-se o Método H;

- Se qc_H < qc_LE, aplica-se o Método LE.

Por fim, foi feita a remoção de dados considerados espúrios, p.ex.: quando a Razão de Bowen foi próxima a -1 , e em seguida pela RBE (RBE $<0$ ou RBE $>2$ ).

\section{Resultados e discussões}

Após a aplicação da filtragem na Razão de Bowen, a porcentagem de dados válidos dos fluxos turbulentos foi de $94,6 \%$. E, da filtragem pela RBE, restaram $75,8 \%$ dos dados de $30 \mathrm{~min}$ (13302 pontos) e a RBE média foi 0,59. A partir destes dados, foi feita a análise da incerteza dos fluxos pelos quatro métodos de pós- fechamento. O Método Combinado diversifica o método de correção pela qualidade do fluxo instantâneo, para verificar a frequência de ocorrência de cada método apresenta-se a Tabela 1, onde se verifica que $62 \%$ dos fluxos foram corrigidos pelo Método Bowen, 29\% pelo Método LE e menos de $8 \%$ pelo Método H.

\subsection{Ciclo Diurno Médio dos fluxos corrigidos}

A figura 1 mostra o ciclo diurno médio dos fluxos de calor sensível (a) e latente (b) medidos em área de campo nativo durante o período de estudo. Os fluxos turbulentos foram ajustados pelos métodos de pós-fechamento como mencionado. As maiores diferenças entre os fluxos corrigidos e os dados brutos ocorreram no período diurno. Para $\mathrm{H}$, a incerteza máxima dos métodos foi em torno de $100 \mathrm{~W} \mathrm{~m}^{-2}$ (Método $\mathrm{H}$ - Método LE), $\sim 45 \mathrm{~W} \mathrm{~m}^{-2}$ (Método Bowen - Método LE e Combinado LE). Enquanto que em relação ao LE, essa incerteza foi aproximadamente $90 \mathrm{~W} \mathrm{m^{-2 }}$ (Método LE - Método H) e $\sim 60 \mathrm{~W} \mathrm{~m}^{-2}$ (Método Bowen - Método H e Combinado - H).

Analisando os valores ajustados pelo Método Combinado, observa-se que a curva é muito próxima da ajustada pelo Método Bowen ou fica entre as curvas do Método Bowen e Método LE, isto porque no período diurno, $\sim 76 \%$ dos dados foram corrigidos pelo Método Bowen e em seguida, pelo Método LE. À noite, em geral, posicionam-se entre as duas curvas porque a porcentagem de dados ajustados por cada método foi dividido quase que igualmente, conforme pode ser visto na Tabela 1.

As figuras 2 e 3 também representam o ciclo diurno dos fluxos corrigidos de $\mathrm{H}$ para a estação do verão (Fig. 2 a) e inverno (Fig. 2 b) e LE referente ao verão (Fig. 3 a) e inverno (Fig. 3 b). Nota-se uma pequena queda nos valores dos fluxos corrigidos de $\mathrm{H}$ do verão (Fig. 2a) para o inverno (Fig. 2b). A incerteza máxima é de $~ 110$ (90) $\mathrm{W} \mathrm{m}^{2}$ no verão (inverno) pelos métodos $\mathrm{H}$ LE e $\sim 45 \mathrm{~W} \mathrm{~m}^{-2}$ nas duas estações pela diferença entre os métodos Bowen - LE e Combinado LE. 
No entanto, quanto aos valores corrigidos de LE (Fig. 3), verifica-se uma queda considerável no inverno por todos os métodos de pósfechamento utilizados neste trabalho. Essa diminuição chegou a ser duas vezes menor em relação ao verão. Tal queda acentuada em LE no inverno pode estar relacionada ao período em que a vegetação entra em estado de senescência e, por conseguinte, diminui sua atividade metabólica. Quanto à incerteza dos métodos, foi de 100 (80) $\mathrm{W} \mathrm{m}^{-2}$ no verão (inverno) pelos métodos LE - H e 70 (40) $\mathrm{W} \mathrm{m}^{-2}$ pelos métodos Bowen - $\mathrm{H}$ e Combinado - $\mathrm{H}$.

Assim, foi verificado que a incerteza dos fluxos pelos métodos de pós-fechamento varia de acordo com o método aplicado. A banda de incerteza obtida pelo Método $\mathrm{H}$ para a correção de $\mathrm{H}$ foi maior do que pelos outros métodos, assim como pelo Método LE para a correção de LE. Quanto ao Método Bowen e Combinado ocorre um decréscimo na banda de incerteza.

Tabela 1: Procedimento realizado para a escolha do método de correção para o Método Combinado e a frequência de ocorrência para o dia completo e por turno, em cada caso.

\begin{tabular}{|c|c|c|c|c|}
\hline Teste & $\begin{array}{c}\text { Método de } \\
\text { Correção }\end{array}$ & $\begin{array}{l}\text { Frequência de } \\
\text { ocorrência (\%) }\end{array}$ & $\begin{array}{r}\text { Frequênci } \\
t\end{array}$ & $\begin{array}{l}\text { orrência por } \\
\text { \%) }\end{array}$ \\
\hline \multirow{2}{*}{ qc_H $=$ qc_LE } & \multirow{2}{*}{ Bowen } & \multirow{2}{*}{61,98} & 75,64 & Diurno \\
\hline & & & 41,57 & Noturno \\
\hline \multirow{2}{*}{ qc_H $>$ qc_LE } & \multirow{2}{*}{$\mathrm{H}$} & \multirow{2}{*}{7,96} & 7,17 & Diurno \\
\hline & & & 9,61 & Noturno \\
\hline \multirow{2}{*}{ qc_H $<$ qc_LE } & \multirow{2}{*}{ LE } & \multirow{2}{*}{28,60} & 17,20 & Diurno \\
\hline & & & 48,82 & Noturno \\
\hline
\end{tabular}
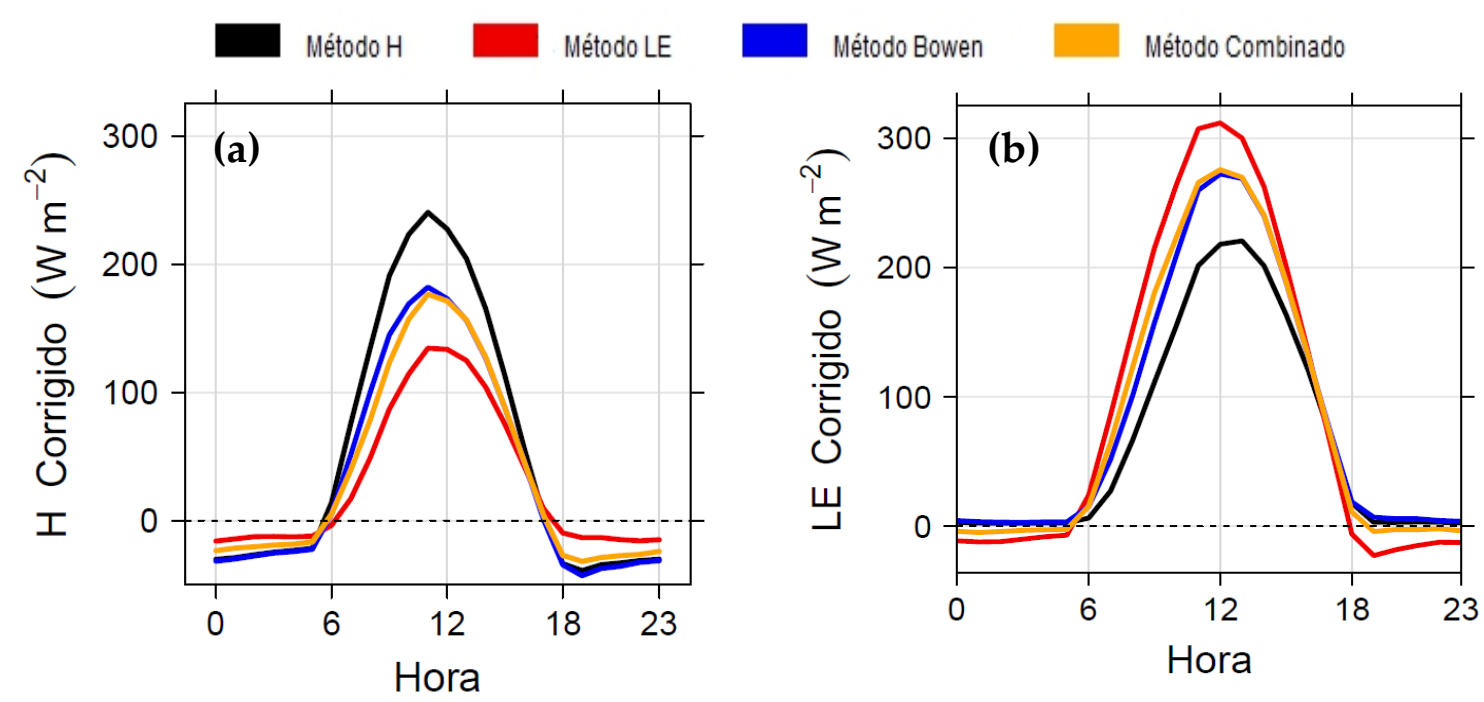

Figura 1: Ciclo Diurno Médio dos Fluxos de Calor: (a) Sensível (H) e (b) Latente (LE) ajustados por quatro métodos de pós-fechamento para o período de 20/11/2013 a 14/12/2014 na área experimental localizada em Pampa brasileiro. 


\subsection{Correção da Evapotranspiração (ET)}

A série temporal do acumulado diário da evapotranspiração bruta e ajustada pelo Método Combinado é representada na figura 4. A ET corrigida é sempre maior que o valor bruto. Assim como o LE diminui significativamente do verão para o inverno, ocorre o mesmo com a ET. A grande variabilidade da ET acompanha também a disponibilidade de energia, ou seja no verão a
ET é alta e no inverno diminui, não apenas pela menor disponibilidade de energia, mas também pela fase de senescência da vegetação. $O$ valor máximo da ET ocorreu no mês de Janeiro, sendo a ET bruta de $5,99 \mathrm{~mm} \mathrm{~d}^{-1}$ e a ET corrigida de $7,19 \mathrm{~mm} \mathrm{~d}^{-1}$. Em média, a ET bruta foi de 2,05 $\mathrm{mm} \mathrm{d}^{-1}$, enquanto que a ET corrigida alcançou 2,58 $\mathrm{mm} \mathrm{d}^{-1}$, uma diferença de 0,53 $\mathrm{mm} \mathrm{d}^{-1}$.
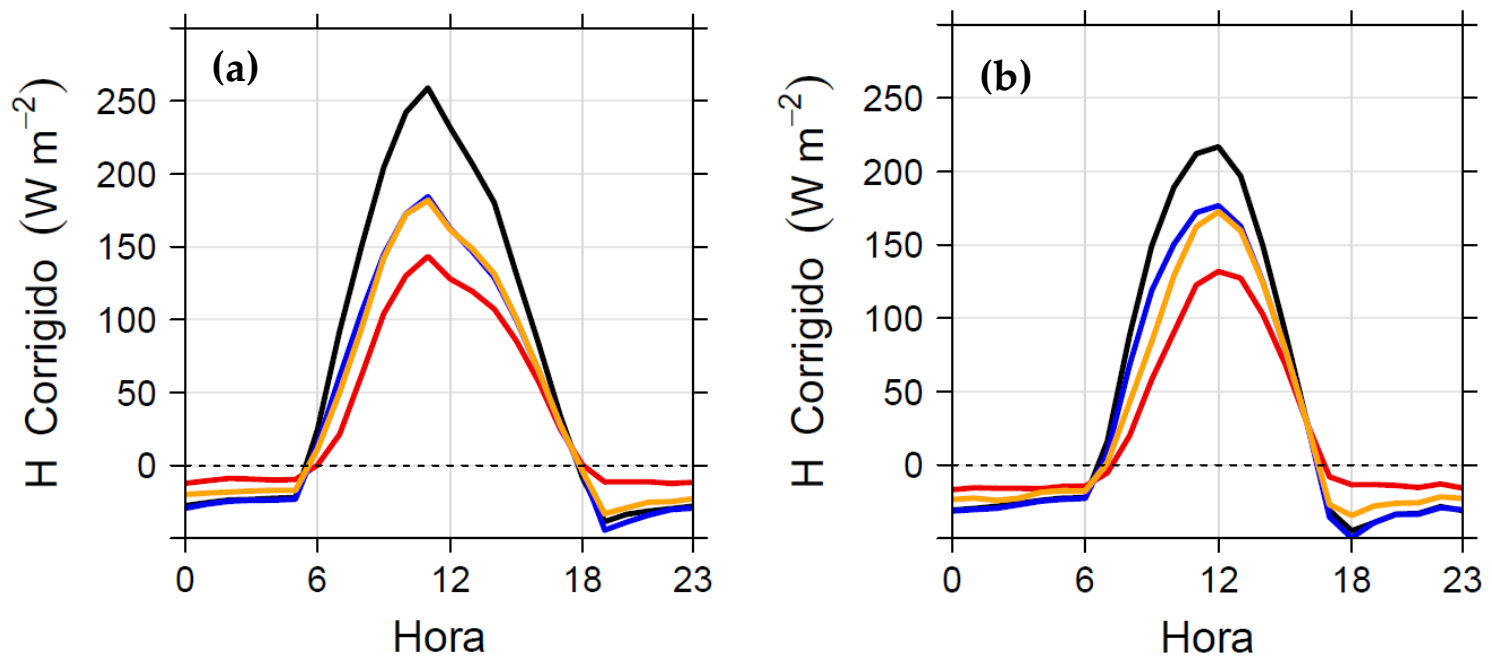

Figura 2: Ciclo Diurno Médio do Fluxo de Calor Sensível $(\mathrm{H})$ corrigido pelos quatro métodos de pósfechamento para as estações: (a) do verão (21/12/2013 a 21/03/2014) e (b) inverno (21/06/2014 a 23/09/2014) para a área experimental localizada em Pampa brasileiro. A identificação de cada método segue a da figura 1.
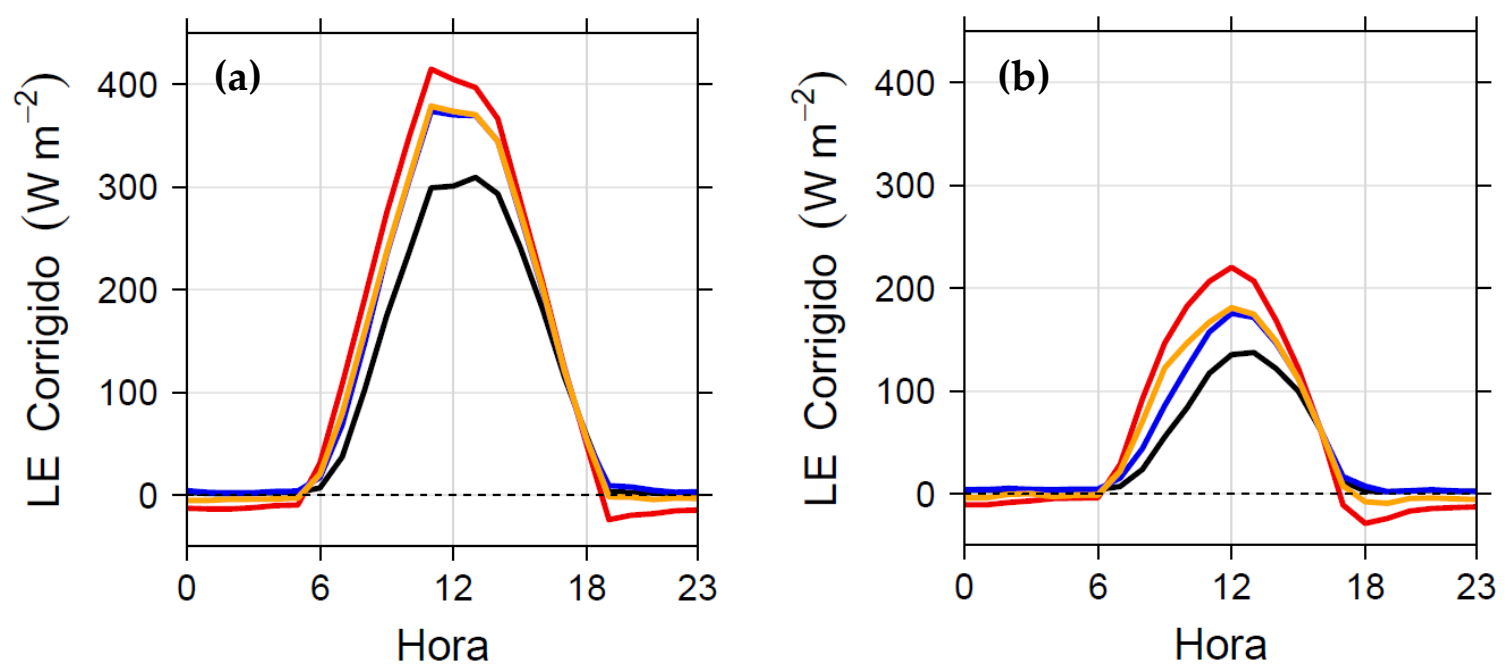

Figura 3: Ciclo Diurno Médio do Fluxo de Calor Latente (LE) corrigido pelos quatro métodos de pósfechamento para as estações: (a) do verão (21/12/2013 a 21/03/2014) e (b) inverno (21/06/2014 a 23/09/2014) para a área experimental localizada em Pampa brasileiro. A identificação de cada método segue a da figura 1. 


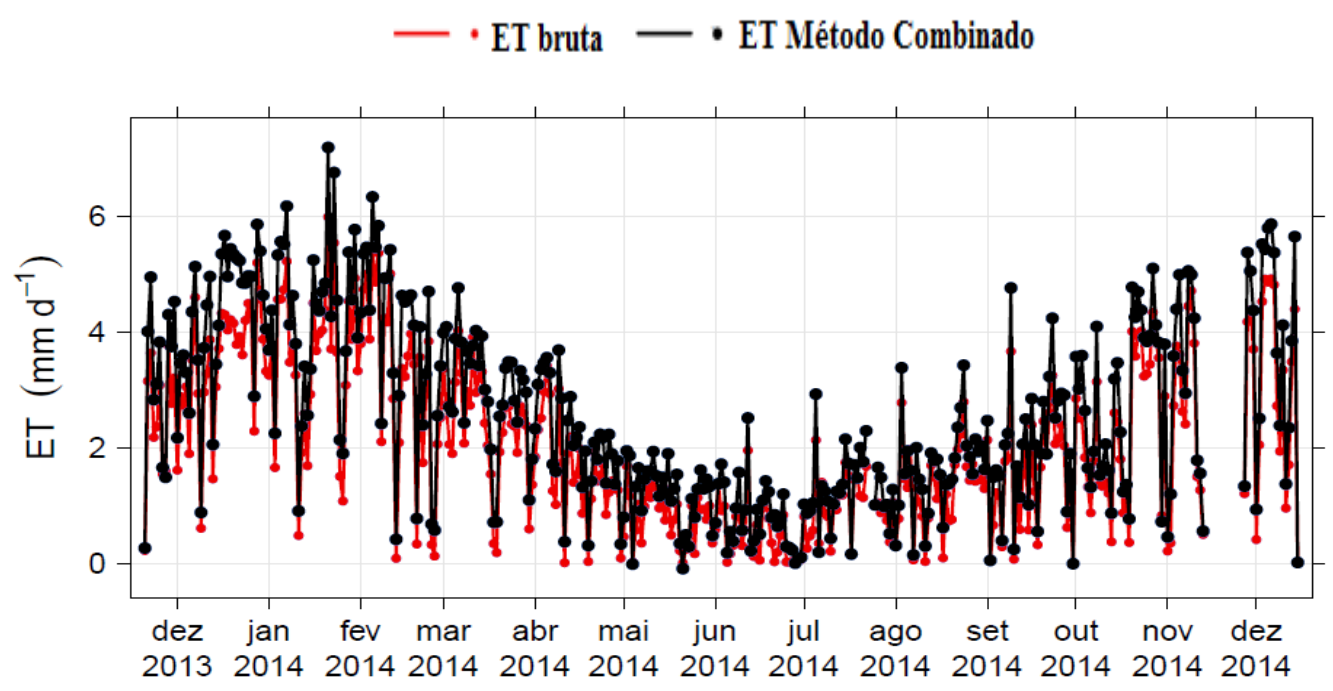

Figura 4: Variação diária acumulada da ET com dados brutos (curva vermelha) e dados corrigidos pelo Método Combinado (curva preta), proposto neste trabalho, para o período de 20/11/2013 a 14/12/2014.

\section{Conclusões}

Conclui-se que o Método de pósfechamento sugerido neste trabalho fornece uma série de fluxos observados corrigidos mais criteriosa uma vez que define o método de correção dos fluxos baseado na qualidade dos fluxos de $\mathrm{H}$ e LE. Essa série de fluxos única (ao invés de uma banda) é essencial para avaliação de erros de modelos de superfície em procedimentos de calibração e validação dos fluxos de energia simulados.

\section{Agradecimentos}

Os autores agradecem à FAPERGS (Proj. 1988-2551/13-3), ao CNPQ (Proj. 461130/20149) e à CAPES pelo financiamento parcial do projeto e das bolsas.

\section{Referências}

Baldocchi, D.D., Hincks, B.B., Meyers, T.P., (1988). Measuring biosphere-atmosphere exchanges of biologically related gases with micrometeorological methods. Ecology 69 (5), 1331-1340.
Foken, T., Göckede, M., Mauder, M., Mahrt, L., Amiro, BD., Munger, JW. (2004) Post-field data quality control. In: X Lee, WJ Massman, B Law (Editors), Handbook of Micrometeorology: A Guide for Surface Flux Measurement and Analysis. Kluwer, Dordrecht, pp. 181-208.

Foken, T., Aubinet, M., Finnigan, J.J., Leclerc, M.Y., Mauder, M., Paw U, K.T., (2011). Results of a panel discussion about the energy balance closure correction for trace gases. Bull. Am. Meteorol. Soc. 92 (4), ES13-ES18.

Leuning, R., van Gorsel, E., Massman, W.J., Isaac, P.R., (2012). Reflections on the surfasse energy imbalance problem. Agric. Forest Meteorol. 156, 65-74.

Moncrieff, J. B., R. Clement, J. Finnigan, and T. Meyers. (2004). Averaging, detrending and filtering of eddy covariance time series, in Handbook of micrometeorology: a guide for surface flux measurements, eds. Lee, X., W. J. Massman and B. E. Law. Dordrecht: Kluwer Academic, 7-31.

Roberti, D. R. ; Tatsch, J. D. ; Oliveira, P. E. S.; Teichrieb, C. A. ; Moraes, O. L. L. de ;Acevedo, O. C ; Zimermann, H. R. SULFLUX - Rede SulBrasileira de Fluxos Superficiais e mudança 
climáticas: resultados preliminares. Sensoriamento Remoto Aplicado à Agricultura: Resultados Projeto SIMTECO. 1ed.Porto Alegre: Evangraf, 2013, v. 1, p. 11-42.

Twine, T.E., Kustas, W.P., Norman, J.M., Cook, D.R., Houser, P.R., Meyers, T.P., Prueger, J.H., Starks, P.J., Wesely, M.L., (2000). Correcting eddy-covariance flux underestimates over a grassland. Agric. For. Meteorol. 103, 279-300.

Webb, E. K., G. I. Pearman, and R. Leuning. 1980. Correction of flux measurements for density effects due to heat and water vapor transfer. Quarterly Journal of the Royal Meteorological Society, 106: 85-100.

Wilczak, J. M., S. P. Oncley, and S. A. Stage. 2001. Sonic anemometer tilt correction algorithms. Boundary-Layer Meteorology, 99: 127-150.

Wilson, K., Goldstein, A., Falge, E., Aubinet, M., Baldocchi, D., Berbigier, P., Bernhofer, C., Ceulemans, R., Dolman, H., Field, C., Grelle, A., Ibrom, A., Law, B.E., Kowalski, A., Meyers, T., Moncrieff, J., Monson, R., Oechel, W., Tenhunen, J., Valentini, R., Verma, S., (2002). Energy balance closure at FLUXNET sites. Agric. Forest Meteorol. 113 (1-4), 223-243. 